\title{
On normalized Laplacian, degree-Kirchhoff index of the strong prism of the dicyclobutadieno derivative of linear phenylenes
}

\author{
Jia-bao $\mathrm{Liu}^{1}$ and Jiaojiao $\mathrm{Gu}^{2}$ \\ ${ }^{1}$ Anhui Xinhua University \\ ${ }^{2}$ Anhui Jianzhu University South Campus
}

September 25, 2021

\begin{abstract}
Phenylenes network is applied in several fields of chemistry sciences due to its advantages compared to other several columnar networks, recently. This paper aims to introduce a kind of networks which obtained by a family of dicyclobutadieno derivative of linear phenylene chain Ln which is made up of $n$ hexagons and $(n+1)$ quadrangles. Let L2n be the strong prism of the dicyclobutadieno derivative of linear phenylenes Ln. By taking full advantage of the knowleges about the normalized Laplacian spectra, we induce the explicit expressions, with respect to the index $n$, of the multiplicative degree-Kirchhoff index and the number of spanning tree based on the graph L2n.
\end{abstract}

\section{Hosted file}

manuscript .pdf available at https://authorea.com/users/336629/articles/538927-on-normalizedlaplacian-degree-kirchhoff-index-of-the-strong-prism-of-the-dicyclobutadieno-derivativeof-linear-phenylenes 